

\title{
ANÁLISE DAS RELAÇÕES ENTRE A GEOMETRIA URBANA E A ORIENTAÇÃO DAS ABERTURAS NA DISPONIBILIDADE DE ILUMINAÇÃO NATURAL NO AMBIENTE INTERNO'
}

\author{
ANALYSIS OFTHE RELATIONSHIP BETWEEN URBAN GEOMETRY \\ AND ORIENTATION OF OPENINGS ON AVAILABILITY OF INDOOR \\ DAYLIGHTING
}

Andréa Coelho Laranja²

Ricardo Carvalho Cabús ${ }^{3}$

Cristina Engel de Alvarez ${ }^{4}$

Leilani Gomes Correia ${ }^{5}$

\section{Resumo}

Este estudo apresenta uma discussão relacionada à iluminação natural no ambiente interno, sendo o objetivo da pesquisa analisar as relações entre a geometria urbana e a orientação das aberturas na disponibilidade de iluminação natural no ambiente interno. Na geometria urbana, foram usadas as relações entre a largura de via $(L)$ e a altura da edificação obstruidora $(H)$, adotando-se relações de $H=2,0 . L, H=2,5$. $L$ e $H=3,0 . L$. Para as aberturas, foram usadas as orientações norte, sul, leste, oeste, nordeste, sudeste, noroeste e sudoeste. Na análise, foram realizadas comparações dos valores de iluminância média global do ambiente interno, com os intervalos das UDI (useful daylight illuminance). Para isso, os valores de iluminância média global foram gerados por meio de simulação na ferramenta computacional TropLux, em um ambiente de geometria retangular inserido em um cenário urbano na cidade de Vitória, latitude $20^{\circ} 19^{\prime} \mathrm{S}$. As simulações foram realizadas para três tipos de céus-padrão da CIE (Commission Internationale L'aclairage). Como principal resultado, constata-se, para os três tipos de céus analisados, a redução das iluminâncias conforme se aumentam as relações entre $\mathrm{H}$ e L. Para o céu 3 (encoberto), constatou-se que, para todas as relações da geometria urbana e para todas as orientações das aberturas, todos os valores de iluminância encontram-se no intervalo suficiente, mas com necessidade de iluminação complementar. No céu 7 (parcialmente nublado) e no céu 12 (claro), a maioria das iluminâncias encontra-se no intervalo suficiente, com algumas exceções para as orientações das aberturas para sul, sudoeste, oeste e sudeste. Para todos os céus, constatou-se também que as maiores taxas de decaimento da iluminância do ambiente interno ocorrem quando a relação da geometria urbana varia de $\mathrm{H}=2,0$. L para $\mathrm{H}=2,5$. L. Para o céu 7 (parcialmente nublado) e 12 (claro), conforme aumenta a relação entre a 
largura de via e a altura da edificação obstruidora, a variação na orientação da abertura passa a ter cada vez menor influência na iluminância.

Palavras-chave: Iluminação natural; Orientação das aberturas; Largura de via; Altura da edificação obstruidora.

\begin{abstract}
This study presents a discussion related to daylighting in the indoor environment, with the research goal of examining the relationship between urban geometry and the orientation of openings in the availability of daylighting in the indoor environment. The ratio between road width $(L)$ and the height of obstructing buildings $(\mathrm{H})$ was used in urban geometry, adopting the $H=2,0 . L, H=2,5 . L$ and $H=3,0 . L$ ratios. North, South, East, West, Northeast, Southeast, Northwest, and Southwest orientations were used for the openings. The overall average illuminance values of the indoor environment were compared with intervals of UDI (Useful Daylight Illuminance). For that, the overall average illuminance values were generated by simulation using the TropLux program, in a rectangular environment inserted in an urban setting in the city of Vitória, latitude $20^{\circ} 19^{\prime} \mathrm{S}$. Simulations were carried out for three types of sky patterns from CIE (Commission Internationale L'aclairage). It is noted that, for all three analyzed sky types, illuminance was reduced as the ratio between $\mathrm{H}$ and $\mathrm{L}$ increased. For sky type 3 (cloudy), it was revealed that, for all urban geometry ratios for all orientations of the openings, all illuminance values are within a sufficient range but in need of additional lighting. Sky type 3 (partly cloudy) and sky type 12 (clear), most illuminances are in the sufficient range, with a few exceptions observed in openings with South, Southwest, West, and Southeast orientations. The highest rates of the indoor illuminance for all sky types was also found to occur when the urban geometry ratios vary from $\mathrm{H}=2,0 . \mathrm{L}$ to $\mathrm{H}=2,5$. L. For sky type 7 (partly cloudy) and sky type 12 (clear), as the ratio between the width of road and height the obstructing buildings increases, the variation in the orientation of the openings has increasingly less influence on illuminance.
\end{abstract}

Key words: Daylighting; Orientation of openings; Road width; Height of obstructing buildings. 
A literatura tem confirmado a dependência da disponibilidade de iluminação natural no ambiente interno das características do seu entorno. Nikiforiadis e Pitts (2003) contestam a avaliação de trabalhos que analisam a iluminação natural e que não levam em consideração o entorno. Littlefair (1998; 2001), Capeluto (2003), Ünver et al. (2003) e Ratti, Baker e Steemers (2005) enfatizam que, entre as variáveis da geometria urbana que se relacionam com a disponibilidade de iluminação natural no ambiente interno, destacam-se as obstruções.

Moraes e Scarazzato (2003) citam que a verticalização das edificações é questão que dificulta o aproveitamento da iluminação natural no espaço urbano. Li et al. (2006) também ressaltam que a altura das edificações é fator influenciador da disponibilidade de iluminação natural no ambiente interno, enquanto Capeluto (2003) complementa que as edificações obstruidoras altas e alongadas afetam sensivelmente a quantidade de iluminação natural e a sua distribuição no ambiente interno de uma edificação.

As pesquisas também demonstram relações entre a altura da edificação obstruidora e a largura da via com o acesso à iluminação natural para o ambiente interno, nas quais se destacam os estudos de Ng (2005), Kruger e Suga (2007), Araújo e Cabús (2007), sendo que estes últimos destacam que tal relação facilita a aplicação em outras dimensões de cânions urbanos.

Outros estudos, por sua vez, correlacionam a atuação da iluminação natural no ambiente interno vinculado à orientação das aberturas desse ambiente. Ünver et al. (2003) e Capeluto (2003) citam a orientação das aberturas como uma das variáveis de influência da iluminação natural no ambiente interno. Li et al. (2006) caracterizam as janelas como as aberturas essencialmente usadas nos edifícios para permitir a admissão da luz natural para o ambiente interno. Tais aberturas configuram-se nas fachadas, que, por sua vez, em muitas situações, orientam-se conforme as vias. Laranja, Cabús e Gazzaneo (2012) constataram aumentos de $121 \%$ a $501 \%$ de iluminância em aberturas orientadas para norte, respectivamente no céu parcialmente nublado e no céu claro, comparado com a orientação sul.

Com base no alicerce conceitual adotado e considerando a hipótese de que a disponibilidade da iluminação natural no ambiente interno (observadas as características específicas do tipo de céu) depende, dentre outros fatores, da geometria urbana e da orientação das aberturas do ambiente interno, o objetivo desta pesquisa foi analisar a influência da geometria urbana, largura de via e altura da edificação obstruidora na disponibilidade de iluminação natural no ambiente interno, variando-se também para isso a orientação das aberturas. Os resultados possibilitam que as escolhas inerentes aos procedimentos projetuais sejam estabelecidos, também, com base na geometria urbana e nas orientações das aberturas, de forma a possibilitar o melhor aproveitamento da iluminação natural e, consequentemente, menor dispêndio de energia elétrica com iluminação artificial.

A pesquisa também contribui para a reflexão e discussão sobre os aspectos inerentes ao planejamento urbano, especial- 
mente para parcelas onde o traçado viário já está consolidado e a viabilidade de ampliação da qualidade de vida dos usuários das edificações se encontra vinculada ao estabelecimento dos recuos frontal, lateral e de fundos para a obtenção de disponibilidade de iluminação natural dos ambientes internos.

\section{método de trabalho}

Considerando o objetivo previamente definido, adotou-se como principal instrumento de análise as simulações computacionais com base no programa TropLux 6.07 (CABÚS, 2012), sendo o recorte territorial a cidade de Vitória, localizada na latitude $20^{\circ} 19^{\prime}$ sul e longitude $40^{\circ} 20^{\prime}$ oeste.

Foram usados na análise os valores das iluminâncias médias globais dos pontos previamente definidos no ambiente interno, sendo esses percentuais comparados aos intervalos de valores das UDI (useful daylight illuminance). Foram usados nas simulações três tipos de céus-padrão da CIE (Commission Internationale L'aclairage): um céu encoberto, um céu parcialmente nublado e um céu claro (COMMISSION INTERNATIONALE L'ACLAIRAGE, 2003).

Laranja (2010), em estudos realizados para valores da média anual de iluminância interna, define os céus 3 (encoberto), 7 (parcialmente nublado) e 12 (claro) como os que correspondem respectivamente aos valores mínimo, intermediário e máximo de iluminância, sendo essas as condições adotadas para as simulações.

Os instrumentos e métodos seguem detalhados, sendo enfatizadas as características do programa de simulação TropLux, os procedimentos para a avaliação da disponibilidade de iluminação natural no ambiente interno, o recorte para os dias e horários selecionados para as simulações, as características do modelo de ambiente interno adotado, a definição dos pontos de avaliação no ambiente interno, as características da edificação obstruidora os procedimentos para a análise de resultados.

\section{Programa de simulação TropLux}

O TropLux permite a simulação das características da iluminação natural em ambiente interno, sendo possível configurar o céu da localidade em que se insere o ambiente por meio da proposta da CIE (CABÚS, 2012). O TropLux se baseia em conceitos e procedimentos estatísticos: o método Monte CarIo; o método do raio traçado; e o conceito de coeficientes de luz natural (CABÚS, 2005). Cabús (2005, p. 241) explica que o método Monte Carlo é baseado na premissa de que "[...] se a probabilidade de ocorrência de cada evento separado é conhecida, então é possível determinar a probabilidade com que a sequência completa de eventos irá ocorrer". No que diz respeito ao método do raio traçado, Cabús (2005) esclarece que ele possibilita trabalhar com geometrias complexas, o qual consiste na técnica em que os raios de luz (retas) incidirão sobre as superfícies (interceptando os planos) e, por sua vez, serão refletidos em direções que podem ser calculadas. Quanto ao coeficiente de luz natural, ainda de acordo com o 
mesmo autor, os coeficientes de luz propostos por Tregenza e Waters (1983) fazem a relação da iluminância de uma dada superfície de acordo com uma determinada subdivisão do céu. Esse autores também usam a iluminância normal num plano desobstruído conforme essa mesma subdivisão. No caso do TropLux, são usados dois tipos de subdivisão: a proposta pela CIE para o cálculo da componente refletida (que divide o céu em 145 partes), e para o cálculo da componente direta, uma subdivisão com 5221 partes, que traz melhorias na precisão dos resultados da componente direta de acordo com o tamanho angular do Sol (CABÚS, 2005).

\section{Avaliação da disponibilidade de iluminação natural no ambiente interno}

Nesta pesquisa, definiu-se que a disponibilidade de iluminação natural no ambiente interno é caracterizada por sua iluminância, com valores que indiquem a adequação à realização das atividades previstas dentro dos compartimentos. Adotaram-se os valores das UDI (useful daylight illuminances) propostas por Nabil e Mardaljevic (2006) como forma de avaliação da disponibilidade de iluminação natural no ambiente interno. Nabil e Mardaljevic (2006) propõem que valores menores que $100 \mathrm{~lx}$ são considerados insuficientes; valores no intervalo entre 100 Ix e 500 Ix caracterizam-se como suficientes, mas com necessidade de iluminação complementar; valores no intervalo suficiente estão entre 500 lx e 2000 lx; e valores caracterizados como excessivos são maiores que $2000 \mathrm{~lx}$.

Os resultados obtidos também foram comparados à norma da ABNT, NBR5413/82, que estabelece os valores de iluminâncias médias mínimas para iluminação artificial em ambientes internos (ASSOCIAÇÃO BRASILEIRA DE NORMAS TÉCNICAS, 1992). De acordo com a NBR 5413/82, os níveis de iluminâncias médias mínimas em serviço para iluminação artificial para uma residência estão entre 75 e 750 Ix. Para quarto ou sala, os valores encontram-se entre 100 e 750 Ix (ASSOCIAÇÃO BRASILEIRA DE NORMAS TÉCNICAS, 1992). Também foi definido que a iluminação a ser tratada diz respeito à iluminação global, a qual é oriunda de parcela proveniente do Sol, e da parcela refletida.

\section{Dias e horários das simulações}

Considerando a necessidade de ampliação máxima do leque de situações possíveis e diferenciadas, e considerando ainda a exequibilidade de realização abrangente de análises, as simulações foram executadas para todos os dias do ano em 11 horários do dia, nos períodos da manhã e da tarde, a cada hora entre $7 \mathrm{~h}$ e $17 \mathrm{~h}$.

\section{Características do ambiente interno adotado}

As simulações foram realizadas para um modelo de ambiente interno, caracterizado por ambiente de uso prolongado em edificação residencial multipavimento, podendo ser, por exemplo, quarto ou sala. A adoção das principais características do modelo foi de acordo com as especificações cons- 
tantes no Código de Obras de Vitória (VITÓRIA, 1998), bem como na verificação de alta ocorrência de ambientes com geometria e acabamentos semelhantes à adotada. Assim, o modelo de ambiente se caracteriza por ter pé-direito de $2,60 \mathrm{~m}$, largura de 2,60 m e comprimento de 3,85 m. As refletâncias internas adotadas foram: piso $=0,2$; paredes $=0,5$; e teto $=0,85$. O modelo analisado tem abertura na fachada voltada para o exterior e centralizada na parede, sendo composta por vidro liso transparente, com área de abertura de $1,25 \mathrm{~m}^{2}$, largura de 1,14 $\mathrm{m}$ e altura de 1,10 m, correspondente a uma proporção de 1/8 da área do piso do compartimento (VITÓRIA, 1998).

A orientação das aberturas foi simulada no cenário urbano na cidade de Vitória-ES (LAT 20 19' S), considerando a fachada com a abertura perpendicular ao traçado urbano, com as vias orientadas para norte-sul, leste-oeste, nordeste-sudoeste, noroeste-sudeste, sendo então adotadas as orientações das aberturas conforme Quadro 1.

Quadro 1 - Orientações das aberturas de acordo com a orientação da via

\begin{tabular}{|l|l|l|}
\hline Orientação da via & \multicolumn{2}{|l|}{ Aberturas } \\
\hline Norte-sul & Leste & Oeste \\
\hline Leste-oeste & Norte & Sul \\
\hline Nordeste-sudoeste & Noroeste & Sudeste \\
\hline Noroeste-sudeste & Nordeste & Sudoeste \\
\hline
\end{tabular}

Fonte: Elaboração própria.

\section{Pontos de medições no ambiente interno}

Observando-se a tipologia-padrão das edificações residenciais multipavimentos em um cenário urbano na cidade de Vitória, usou-se como padrão o estudo do primeiro pavimento-tipo, que habitualmente localiza-se no quarto andar, considerando o uso dos pavimentos inferiores normalmente serem destinados para lazer e garagem. Adotou-se a norma da ABNT, NBR 15215-4 (ASSOCIAÇÃO BRASILEIRA DE NORMAS TÉCNICAS, 2005), que normaliza a quantidade de pontos de medições para iluminação natural necessários em um compartimento, bem como a localização desses pontos. A norma usa como referência as próprias características do compartimento: comprimento, altura, largura e posição do ponto de referência (local de trabalho, adotado como $75 \mathrm{~cm}$ do piso do ambiente interno). Para que durante a verificação do nível de iluminação natural o erro seja inferior a 10\%, é preciso determinar um número mínimo de pontos. Dessa forma, de acordo com a NBR 15215-4 (ASSOCIAÇÃO BRASILEIRA DE NORMAS TÉCNICAS, 2005), o número mínimo encontrado foi de 16 pontos, optando-se por trabalhar com um número de pontos superior a 16, com um grid com 5 linhas e 5 colunas, totalizando 25 pontos de medições, conforme Figura 1. Conforme as dimensões do ambiente, as distâncias dos pontos são, em um sentido, de 0,43 m entre si, e no outro alinhamento, de 0,64 cm entre si. 


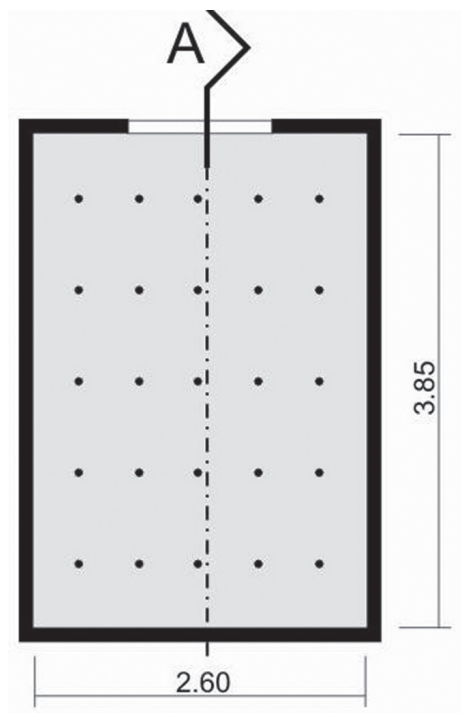

\section{Características da edificação obstruidora}

Adotaram-se as alturas de 36 m, 45 m e 54 m para a edificação obstruidora. A primeira altura de $36 \mathrm{~m}$ corresponde a uma edificação de 12 andares, comumente encontrada no cenário urbano estudado. A partir daí, foram acrescentados mais três andares, obtendo uma altura de $45 \mathrm{~m}$, e, após mais três andares, obtendo uma altura de $54 \mathrm{~m}$, considerando a tendência à verticalização da área em estudo.

No que se refere à largura da via, foram adotadas como objeto das simulações características de vias, de acordo com as tipologias físicas estruturais da rede viária básica do cenário urbano estudado. Dessa forma, tomando-se a tipologia de via "local principal" definida pelo Plano Diretor Urbano de Vitória (VITÓRIA, 2006), adotou-se o valor de $18 \mathrm{~m}$ (rua+passeio) para a simulação.

Dessa forma, a largura de via de $18 \mathrm{~m}$ foi testada com as alturas da edificação obstruidora de 36 m, 45 m e 54 m, o que representa respectivamente as relações de largura de via e altura de edificação obstruidora de $H=2,0 . L, H=2,5 . L$ e $H=3,0 . L$, conforme Figuras 2 e 3.

$$
\mathrm{H}=2,0 . \mathrm{L}
$$

$$
H: 36 \mathrm{~m} \quad \mathrm{~L}: 18 \mathrm{~m}
$$$$
\mathrm{H}=2,5 . \mathrm{L}
$$
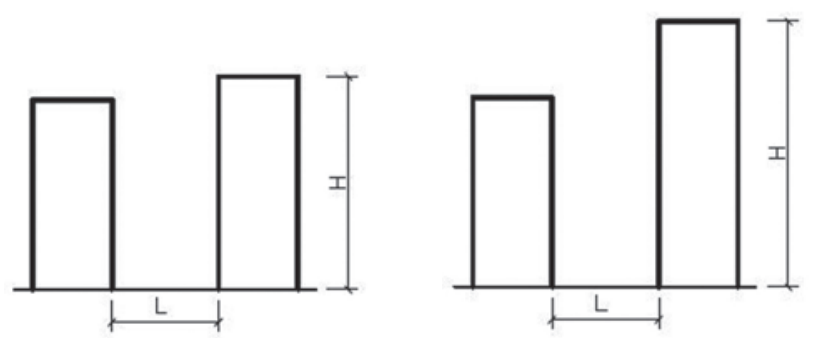

Figura 1 - Planta-baixa e corte, sem escala, com pontos de medição da iluminância. Fonte: Elaboração própria.

Figura 2 • Imagem que demonstra as relações entre L (largura de via) e H (altura da edificação obstruidora) adotadas. Fonte: Elaboração própria..

$$
\mathrm{H}=3,0 . \mathrm{L}
$$

$\mathrm{H}: 54 \mathrm{~m} \quad \mathrm{~L}: 18 \mathrm{~m}$

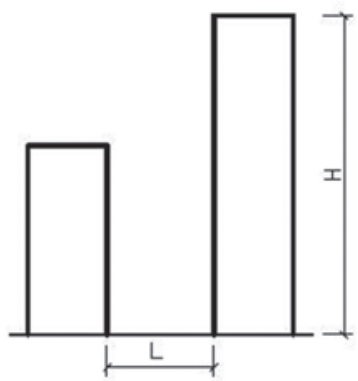

Cadernos de Arquitetura e Urbanismo, v.20, n.2b, $1^{10}$ sem. 2013 

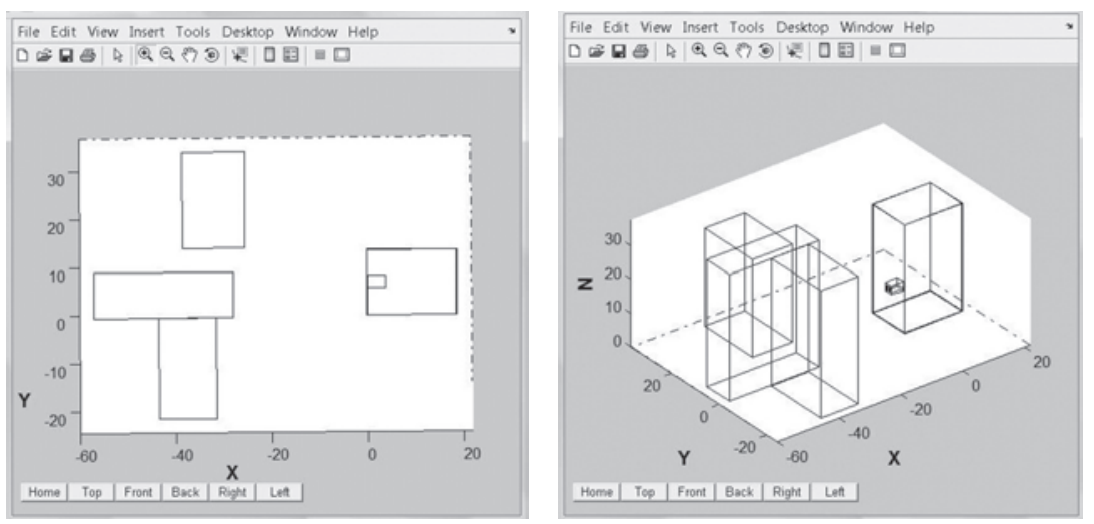

No que se refere à característica relacionada à reflexão das superfícies, é preciso atribuir-lhes um valor que represente sua efetiva capacidade, assim, em razão disso - e adotando como referência o trabalho desenvolvido por Nikiforiadis e Pitts (2003) e Araújo e Cabús (2007) -, foram adotados valores médios que caracterizam, de forma satisfatória e o mais próximo da realidade, a capacidade de reflexão das superfícies externas verticais (edificação obstruidora) e horizontais (vias). Dessa forma, para a realização das características de reflexão dos fechamentos verticais opacos do cenário urbano, foi utilizado como referência o trabalho desenvolvido por $\mathrm{Ng}$ (2005), o qual encontra como refletância média dos fechamentos opacos o valor de $40 \%$. Para o solo, o valor adotado é de acordo com o tipo de pavimentação, o qual, para o recorte, considerando a regulamentação urbana, pode-se usar asfalto, concreto, bloquete ou paralelepípedo. Dessa forma, como refletância média das superfícies horizontais, adotou-se a refletância de $20 \%$.

\section{Análises de resultados}

Os resultados das simulações permitiram a análise dos valores de iluminância média global que se enquadram nos intervalos de valores das UDI, bem como dos níveis de iluminância média mínima, conforme a NBR 5413/82, para quarto ou sala.

Na análise da iluminância interna, objetivou-se verificar para cada relação entre largura de via e altura da edificação obstruidora conforme também a orientação das aberturas: a) se ocorreram alterações na iluminância média global; b) como se comportam as alterações da iluminância média global; c) se há uma tendência a um comportamento semelhante da iluminância média global para as diversas orientações; d) como se enquadram os valores de iluminância média global com relação aos intervalos de valores das UDI; e) como se enquadram os valores de iluminância média global com relação aos valores estabelecidos pela NBR 5413/82.

\section{Resultados}

Considerando a metodologia estabelecida, os resultados foram definidos com base em dois enfoques de análise: o da

Figura $\mathbf{3} \bullet$ Imagens em 3D geradas pelo TropLux, com a vista superior da edificação obstruidora e identificação do ambiente interno analisado. Fonte: Elaboração própria. 
iluminância interna global, conforme as relações entre largura de via e altura da edificação obstruidora; e o da orientação das aberturas, cujo detalhamento segue descrito.

\section{Análises da iluminância interna}

Os resultados obtidos indicam que, para o céu 3 (encoberto), como demonstra a Tabela 1, o aumento da altura da edificação obstruidora acarreta redução nos valores de iluminância para todas as orientações. A variação na orientação das aberturas, porém, não ocasiona alterações nos valores de iluminância. Dessa forma, para o céu 3, somente o aumento na altura da edificação obstruidora contribui para reduções nos valores de iluminância.

Observa-se também que tanto o aumento da altura da edificação obstruidora nas relações $H=2,0 . L, H=2,5 . L$ e $H=3,0 . L$ quanto a variação na orientação das aberturas apresentam todos os valores de iluminância no intervalo entre $100 \mathrm{~lx}$ e $500 \mathrm{Ix}$, caracterizados pelo intervalo das UDI como suficientes, mas com necessidade de iluminação complementar. No que diz respeito à NBR5413/82, para todas as orientações das aberturas, e as relações entre a largura de via e altura da edificação, os valores encontrados estão em consonância com os estabelecidos para quarto e sala, entre 100 e 750 lx.

Tabela 1 - Valor da média anual de iluminância interna conforme $\mathrm{H}$ (altura da edificação obstruidora), L (largura de via) e a orientação da abertura para a condição de céu 3 (encoberto).

\begin{tabular}{l|l|l|l}
\hline \multicolumn{3}{l}{ Iluminância interna (Ix) para céu 3 (encoberto) } \\
\hline \multirow{4}{*}{ Orientação da abertura } & H: $36 \mathrm{~m}$ & H: $45 \mathrm{~m}$ & H: $54 \mathrm{~m}$ \\
\cline { 2 - 4 } & L: $18 \mathrm{~m}$ & $\mathrm{~L}: 18 \mathrm{~m}$ & $\mathrm{~L}: 18 \mathrm{~m}$ \\
\cline { 2 - 4 } & $\mathrm{H}=2,0 . \mathrm{L}$ & $\mathrm{H}=2,5 . \mathrm{L}$ & $\mathrm{H}=3,0 . \mathrm{L}$ \\
\hline Todas & 220,9 & 182,7 & 160,3 \\
\hline
\end{tabular}

Fonte: Elaboração própria.

Para o céu 3 (encoberto), a Tabela 2 mostra que, para todas as orientações, as maiores reduções percentuais da iluminância ocorrem quando a altura da edificação obstruidora aumenta de $36 \mathrm{~m}$ (relação $H=2,0 . L$ ) para $45 \mathrm{~m}$ (relação $H=2,5 . L$ ). Essa redução percentual é cerca de 1,5 vezes superior quando comparada com o aumento da edificação obstruidora de $45 \mathrm{~m}$ (relação $\mathrm{H=2,5.L \text {) }}$ para $54 \mathrm{~m}$ (relação $\mathrm{H=3}, 0 . \mathrm{L}$ ). Assim, para o céu 3 (encoberto), a variação nas relações entre altura de edificação obstruidora $(H)$ e largura de via $(L)$ de $H=2,0$. L para $H=2,5$. L compreendem maiores taxas de decaimento da iluminância do ambiente interno, e a variação nas relações de $\mathrm{H}=2,5 . \mathrm{L}$ para $\mathrm{H}=3,0$. $\mathrm{L}$ compreendem menores taxas de decaimento da iluminância. Portanto a atenção deve estar voltada para as relações com maiores taxas de decaimento, considerando serem as de maior interferência na disponibilidade de iluminação natural no ambiente interno.

Para o céu 7 (parcialmente nublado), a Tabela 3 mostra que o aumento da altura da edificação obstruidora acarreta redução nos valores de iluminância para todas as orientações. Observa-se também que a variação na orientação das aberturas também 
Tabela 2 - Percentual de redução de iluminância interna

conforme a altura da edificação obstruidora para a condição de céu 3 (encoberto).

\section{Percentual de redução de iluminância (\%) para céu 3 (encoberto)}

\begin{tabular}{l|l|l}
\hline \multirow{2}{*}{$\begin{array}{l}\text { Orientação da } \\
\text { abertura }\end{array}$} & $\begin{array}{l}\text { Aumento da altura da edificação obstruidora } \\
\text { de } 36 \mathrm{~m} \text { para } 45 \mathrm{~m}\end{array}$ & $\begin{array}{l}\text { Aumento da altura da edificação obstruidora } \\
\text { de } 45 \mathrm{~m} \text { para } 54 \mathrm{~m}\end{array}$ \\
\cline { 2 - 3 } & Largura de via: $18 \mathrm{~m}$ & Largura de via: $18 \mathrm{~m}$ \\
\hline Todas & $17 \%$ & $12 \%$ \\
\hline
\end{tabular}

Fonte: Elaboração própria.

contribui para alteração nos valores de iluminância, em que as maiores diferenças entre os valores de iluminância ocorrem na altura de edificação obstruidora de 36 m (relação $H=2$. L), decaindo os valores conforme aumenta a altura da edificação obstruidora.

No que diz respeito à NBR5413/82, para todas as orientações das aberturas, e as relações entre a largura de via e altura da edificação analisados, os valores encontrados estão em consonância com os estabelecidos para quarto e sala, entre 100 e 750 Ix. As exceções ocorrem na altura de edificação obstruidora de $36 \mathrm{~m}$ (relação $\mathrm{H}=2$.L), nas orientações noroeste, norte, nordeste, leste, onde os valores apresentam-se superiores ao valor mais alto estipulado pela NBR5413/82. Esse resultado indica a necessidade do uso de proteções solares, o qual reduzirá a iluminação natural no ambiente interno, com benefícios, porém, na redução do efeito de ofuscamento.

Tabela 3 - Valor da média anual de iluminância interna conforme $\mathrm{H}$ (altura da edificação obstruidora), L (largura de

via)e a orientação da abertura para a condição de céu 7 (parcialmente nublado).

\begin{tabular}{l|l|l|l}
\hline Iluminância interna (Ix) para céu 7 (parcialmente nublado) \\
\hline \multirow{2}{*}{ Orientação da abertura } & H: $36 \mathrm{~m}$ & H: $45 \mathrm{~m}$ & H: $54 \mathrm{~m}$ \\
\cline { 2 - 4 } & L: $18 \mathrm{~m}$ & L: $18 \mathrm{~m}$ & L: $18 \mathrm{~m}$ \\
\cline { 2 - 4 } & $\mathrm{H}=2,0 . \mathrm{L}$ & $\mathrm{H}=2,5 . \mathrm{L}$ & $\mathrm{H}=3,0 . \mathrm{L}$ \\
\hline \multirow{2}{*}{ Sul } & 441,9 & 391 & 361,4 \\
\hline Sudoeste & 552,2 & 470 & 424,7 \\
\hline Oeste & 737,1 & 582,9 & 488,3 \\
\hline Noroeste & 855,3 & 668,7 & 550,3 \\
\hline Norte & 905,8 & 637,5 & 521,6 \\
\hline Nordeste & 844,5 & 686,4 & 583,5 \\
\hline Leste & 766,6 & 619,4 & 551,4 \\
\hline Sudeste & 579,5 & 517,1 & 447,9 \\
\hline Diferença entre o maior e o menor valor de iluminância & 463,1 & 295,4 & 221,1 \\
\hline
\end{tabular}

Fonte: Elaboração própria.

Ressalta-se que, para cada relação da geometria urbana $(H$ e $L)$, observada a orientação, vão sendo reduzidas as diferenças de valores entre a maior e a menor iluminância, ou seja, a orientação da abertura tem maior influência na iluminância na altura de edificação obstruidora de $36 \mathrm{~m}$ (relação $\mathrm{H}=2$. L), e conforme cresce a relação para $H=2,5$. $L$ e $H=3,0 . L$, a variação na orientação da abertura passa a ter cada vez menor influência na iluminância. 
Observa-se também na Tabela 3 que, para a altura de edificação obstruidora de $36 \mathrm{~m}$ (relação $\mathrm{H}=2$.L), os valores de iluminância apresentam uma progressão de aumento que tem o valor máximo na orientação de abertura para Norte. Para as alturas da edificação obstruidora de $45 \mathrm{~m}$ (relação $\mathrm{H=2,5}$. L) e $54 \mathrm{~m}$ (relação $H=3,0$.L) os valores de iluminância apresentam uma progressão de aumento que tem os maiores valores na orientação Nordeste.

Na relação $H=2,0 . L$, com exceção da orientação da abertura para Sul, todas as orientações de abertura apresentam os valores de iluminância no intervalo entre 500 lx e 2.000 Ix, caracterizados como suficientes; o mesmo se verificando na relação $\mathrm{H}=2,5 . \mathrm{L}$, com exceção da orientação da abertura para sul e sudoeste. Já na relação $\mathrm{H}=3,0 . \mathrm{L}$, com exceção da orientação da abertura para sul, sudoeste, oeste e sudeste, as demais orientações de abertura apresentam os valores de iluminância no intervalo entre 500 e $2.000 \mathrm{~lx}$, caracterizados como suficientes.

Para o céu 7 (parcialmente nublado), a Tabela 4 mostra que, para todas as orientações, com exceção da sudeste, as maiores reduções percentuais da iluminância ocorrem quando a altura da edificação obstruidora aumenta de $36 \mathrm{~m}$ (relação $H=2$. L) para $45 \mathrm{~m}$ (relação $H=2,5 . L$ ). Dessa forma, para o céu 7 (parcialmente nublado), a variação nas relações entre altura de edificação obstruidora $(H)$ e largura de via $(L)$ de $H=2,0 . L$ para $\mathrm{H}=2,5 . \mathrm{L}$ compreende maiores taxas de decaimento da iluminância do ambiente interno, enquanto a variação nas relações de $H=2,5 . L$ para $H=3,0 . L$ compreende menores taxas de decaimento da iluminação natural no ambiente interno. Portanto a atenção deve estar voltada para as relações com maiores taxas de decaimento, visto serem as de maior interferência na disponibilidade de iluminação natural no ambiente interno.

Para o céu 12 (claro), de acordo com a Tabela 5, o aumento da altura da edificação obstruidora acarretou redução nos valores de iluminância, fato este que ocorreu para quase todas as orientações das aberturas.

Tabela 4 - Percentual de redução de iluminância interna conforme a altura da edificação obstruidora para a condição de céu 7 (parcialmente nublado).

\begin{tabular}{l|l|l}
\hline \multicolumn{2}{l}{ Percentual de redução de lluminância (\%) para céu 7 (parcialmente nublado) } \\
\hline \multirow{2}{*}{$\begin{array}{l}\text { Orientação da } \\
\text { abertura }\end{array}$} & $\begin{array}{l}\text { Aumento da altura da edificação } \\
\text { obstruidora de } 36 \mathrm{~m} \text { para } 45 \mathrm{~m}\end{array}$ & $\begin{array}{l}\text { Aumento da altura da edificação } \\
\text { obstruidora de } 45 \mathrm{~m} \text { para } 54 \mathrm{~m}\end{array}$ \\
\cline { 2 - 3 } & Largura de via $18 \mathrm{~m}$ & Largura de via $18 \mathrm{~m}$ \\
\hline Sul & $-12 \%$ & $-8 \%$ \\
\hline Sudoeste & $-15 \%$ & $-10 \%$ \\
\hline Oeste & $-21 \%$ & $-16 \%$ \\
\hline Noroeste & $-22 \%$ & $-18 \%$ \\
\hline Norte & $-30 \%$ & $-18 \%$ \\
\hline Nordeste & $-19 \%$ & $-15 \%$ \\
\hline Leste & $-19 \%$ & $-11 \%$ \\
\hline Sudeste & $-11 \%$ & $-13 \%$ \\
\hline
\end{tabular}


Observa-se que, nas relações $H=2,0 . L, H=2,5 . L, H=3,0 . L$, com exceção da orientação da abertura para sul, todas as orientações de abertura apresentam os valores de iluminância no intervalo entre $500 \mathrm{~lx}$ e $2.000 \mathrm{~lx}$, caracterizados como suficientes. Na orientação Sul nas relações $H=2,0 . L, H=2,5 . L$, $H=3,0 . L$, os valores de iluminância encontram-se no intervalo entre $100 \mathrm{~lx}$ e $500 \mathrm{~lx}$, caracterizados como suficientes, mas com necessidade de iluminação complementar.

No que diz respeito à NBR5413/82, somente para as orientações sul e sudoeste nas relações $H=2,0 . L, H=2,5 . L, H=3,0 . L$, os valores encontrados estão em consonância com os estabelecidos para quarto e sala, entre 100 e 750 lx. Para as demais orientações, nas relações de altura $H=2,0 . L, H=2,5 . L$, $H=3,0 . L$, os valores apresentam-se superiores ao índice mais alto estipulado pela NBR 5413/82. Esse resultado indica a necessidade do uso de proteções solares, o qual reduzirá a iluminação natural no ambiente interno, com benefícios, porém, na redução do efeito de ofuscamento.

Tabela 5 - Valor da média anual de iluminância interna conforme $\mathrm{H}$ (altura da edificação obstruidora), L (largura de via) e orientação da abertura para a condição de céu 12 (claro).

\begin{tabular}{|c|c|c|c|}
\hline \multicolumn{4}{|l|}{ Iluminância interna (Ix) para céu 12 (claro) } \\
\hline \multirow{3}{*}{ Orientação da abertura } & $\mathrm{H}: 36 \mathrm{~m}$ & $\mathrm{H}: 45 \mathrm{~m}$ & $\mathrm{H}: 54 \mathrm{~m}$ \\
\hline & L: $18 \mathrm{~m}$ & $\mathrm{~L}: 18 \mathrm{~m}$ & $\mathrm{~L}: 18 \mathrm{~m}$ \\
\hline & $\mathrm{H}=2,0 . \mathrm{L}$ & $\mathrm{H}=2,5 . \mathrm{L}$ & $\mathrm{H}=3,0 . \mathrm{L}$ \\
\hline Sul & 374,8 & 374,5 & 385 \\
\hline Sudoeste & 719,9 & 637 & 599,6 \\
\hline Oeste & 1224,4 & 939,6 & 769,2 \\
\hline Noroeste & 1553,2 & 1174,5 & 932,7 \\
\hline Norte & 1752,1 & 1049,5 & 823,2 \\
\hline Nordeste & 1503,6 & 1229,1 & 1049 \\
\hline Leste & 1343,2 & 1074,2 & 986,4 \\
\hline Sudeste & 803 & 796,5 & 680,7 \\
\hline $\begin{array}{l}\text { Diferença entre o maior e o menor valor de } \\
\text { iluminância }\end{array}$ & 1377,3 & 854,6 & 601,4 \\
\hline
\end{tabular}

Fonte: Elaboração própria.

Ressalta-se que para cada relação da geometria urbana $(H$ e $L)$, observada a orientação, percebe-se a redução das diferenças de valores entre a maior e a menor iluminância, ou seja, a orientação da abertura tem maior influência na iluminância na altura de edificação obstruidora de $36 \mathrm{~m}$ (relação $\mathrm{H}=2 . \mathrm{L}$ ), e conforme cresce a relação para $H=2,5 . L$ e $H=3,0$. L, a variação na orientação da abertura passa a ter cada vez menor influência.

Para o céu 12 (claro), a Tabela 6 mostra que, para as orientações norte, as maiores reduções percentuais da iluminância ocorrem quando a altura da edificação obstruidora eleva-se de $36 \mathrm{~m}$ para $45 \mathrm{~m}$. Quando o aumento da altura das edificações 
ocorre da altura $45 \mathrm{~m}$ para $54 \mathrm{~m}$, a redução percentual diminui para cerca da metade do valor.

Tabela 6 - Percentual de redução de iluminância interna conforme a altura da edificação obstruidora para a condição de céu 12 (claro).

\begin{tabular}{l|l|l}
\hline \multicolumn{2}{l}{ Percentual de redução de iluminância (\%) para céu 12 (claro) } \\
\hline \multirow{2}{*}{$\begin{array}{l}\text { Orientação da } \\
\text { abertura }\end{array}$} & $\begin{array}{l}\text { Aumento da altura da edificação } \\
\text { obstruidora de } 36 \mathrm{~m} \text { para } 45 \mathrm{~m}\end{array}$ & $\begin{array}{l}\text { Aumento da altura da edificação } \\
\text { obstruidora de } 45 \mathrm{~m} \text { para } 54 \mathrm{~m}\end{array}$ \\
\cline { 2 - 3 } & Largura de via $18 \mathrm{~m}$ & Largura de via $18 \mathrm{~m}$ \\
\hline Sul & $0 \%$ & $3 \%$ \\
\hline Sudoeste & $-12 \%$ & $-6 \%$ \\
\hline Oeste & $-23 \%$ & $-18 \%$ \\
\hline Noroeste & $-24 \%$ & $-21 \%$ \\
\hline Norte & $-40 \%$ & $-22 \%$ \\
\hline Nordeste & $-18 \%$ & $-15 \%$ \\
\hline Leste & $-20 \%$ & $-8 \%$ \\
\hline Sudeste & $-1 \%$ & $-15 \%$ \\
\hline
\end{tabular}

Fonte: Elaboração própria.

Dessa forma, o aumento da edificação obstruidora de $36 \mathrm{~m}$ para $45 \mathrm{~m}$ causa uma maior redução percentual do que o aumento da edificação obstruidora de $45 \mathrm{~m}$ para $54 \mathrm{~m}$. Nos dois casos, o aumento da edificação obstruidora de $36 \mathrm{~m}$ para $45 \mathrm{~m}$ e de $45 \mathrm{~m}$ para $54 \mathrm{~m}$ obteve as maiores reduções percentuais de iluminância na orientação de abertura para norte. Percebe-se que, conforme as orientações das aberturas aproximam-se da orientação norte, aumenta-se o percentual de redução das iluminâncias.

Com base nos resultados obtidos, a Tabela 7 apresenta uma proposta considerando cada condição de céu, a relação da geometria urbana $(\mathrm{H}$ e $\mathrm{L}$ ) e a orientação da abertura, para que sejam mantidas as iluminâncias internas dentro do intervalo suficiente das UDI: $100 \mathrm{~lx} \leq \mathrm{UDI}<500 \mathrm{~lx}$ (suficiente, mas com necessidade de iluminação complementar), $500 \mathrm{~lx} \leq \mathrm{UDI}<2000 \mathrm{~lx}$ (suficiente).

\section{Conclusão}

Com base nos resultados obtidos, foi possível verificar a influência da geometria urbana e da orientação das aberturas na disponibilidade de iluminação natural no ambiente interno, sendo perceptíveis as alterações nos valores de iluminância interna global, bom base nas alterações nas relações entre a largura de via, altura da edificação obstruidora e orientação das aberturas.

Para o céu 3 (encoberto), que não sofre a influência da orientação, constatou-se que os valores de iluminância reduzem-se conforme aumentam as relações entre $\mathrm{H}$ e L. Para o céu 7 (parcialmente nublado) e céu 12 (claro), o aumento da altura da edificação obstruidora também acarretou na redução dos valores de iluminância, quando a relação da geometria urbana varia de $H=2,5 . L$ e $H=3,0 . L$. 
Tabela 7 - Identificação das relações entre $\mathrm{H}$ e L, observadas a orientação das aberturas e condição de céu, para que sejam mantidas as médias anuais de iluminância interna nos intervalos suficientes das UDI: 100 Ix $\leq$ UDI <500

Ix (suficiente, mas com necessidade de iluminação complementar), 500 Ix $\leq$ UDI <2 000 Ix (suficiente).

\begin{tabular}{|c|c|c|c|c|c|c|c|c|c|c|c|c|c|c|c|c|c|c|}
\hline \multirow{3}{*}{$\begin{array}{l}\text { Orientação da } \\
\text { abertura }\end{array}$} & \multicolumn{9}{|c|}{$\begin{array}{l}100 \leq \mathrm{UDI}<500 \mathrm{Ix} \\
\text { (suficiente, mas com necessidade de ilumi- } \\
\text { nação complementar) }\end{array}$} & \multicolumn{9}{|c|}{$\begin{array}{l}500 \leq \mathrm{UDI}<2000 \mathrm{~lx} \\
\text { (suficiente) }\end{array}$} \\
\hline & \multicolumn{3}{|c|}{ Céu 3} & \multicolumn{3}{|c|}{ Céu 7} & \multicolumn{3}{|c|}{ Céu 12} & \multicolumn{3}{|c|}{ Céu 3} & \multicolumn{3}{|c|}{ Céu 7} & \multicolumn{3}{|c|}{ Céu 12} \\
\hline & $\begin{array}{l}\text { ب! } \\
\text { i⿱ } \\
\text { II }\end{array}$ & $\begin{array}{l}\text { Li⿱ } \\
\text { II } \\
\text { I }\end{array}$ & 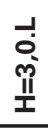 & $\begin{array}{l}\text { à } \\
\text { Ni } \\
\text { II }\end{array}$ & 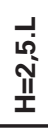 & 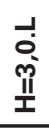 & $\begin{array}{l}\text { ب0 } \\
\text { Ṅ } \\
\text { II }\end{array}$ & กุ่ & $\begin{array}{l}\text { ¿̊́ } \\
\text { II } \\
\text { II }\end{array}$ & $\begin{array}{l}\text { ب. } \\
\stackrel{0}{N} \\
\text { II }\end{array}$ & 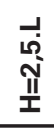 & 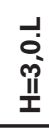 & 모 & 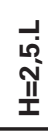 & 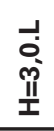 & $\begin{array}{l}\text { ㅁ } \\
\text { Ñ } \\
\text { II }\end{array}$ & เุ & $\begin{array}{l}0 \\
0 \\
\text { II } \\
\text { I }\end{array}$ \\
\hline Sul & $x$ & $x$ & $x$ & $x$ & $x$ & $x$ & $x$ & $x$ & $x$ & & & & & & & & & \\
\hline Sudoeste & $x$ & $\mathrm{x}$ & $x$ & & $x$ & $x$ & & & & & & & $x$ & & & $x$ & $x$ & $x$ \\
\hline Oeste & $x$ & $x$ & $x$ & & & $x$ & & & & & & & $x$ & $x$ & & $x$ & $x$ & $\mathrm{x}$ \\
\hline Noroeste & $x$ & $x$ & $x$ & & & & & & & & & & $x$ & $x$ & $x$ & $x$ & $x$ & $\mathrm{x}$ \\
\hline Norte & $x$ & $\mathrm{x}$ & $x$ & & & & & & & & & & $x$ & $\mathrm{x}$ & $x$ & $x$ & $x$ & $\mathrm{x}$ \\
\hline Nordeste & $x$ & $x$ & $x$ & & & & & & & & & & $x$ & $x$ & $x$ & $x$ & $x$ & $\mathrm{x}$ \\
\hline Leste & $x$ & $x$ & $x$ & & & & & & & & & & $x$ & $x$ & $x$ & $x$ & $x$ & $\mathrm{x}$ \\
\hline Sudeste & $x$ & $x$ & $x$ & & & $x$ & & & & & & & $x$ & $x$ & & $x$ & $x$ & $\mathrm{x}$ \\
\hline
\end{tabular}

Fonte: Elaboração própria.

Para o céu 3 (encoberto), constatou-se que, em todas as relações da geometria urbana $(H=2,0 . L, H=2,5$. $L$ e $H=3,0 . L)$, para todas as orientações das aberturas, todos os valores de iluminância encontram-se no intervalo suficiente das UDI, mas com necessidade de iluminação complementar. No que diz respeito ao céu 7 (parcialmente nublado), para todas as relações ( $H=2,0 . L, H=2,5 . L, H=3,0 . L)$, com exceção da orientação da abertura para sul (nas relações $H=2,0 . L, H=2,5 . L, H=3,0 . L$ ), sudoeste (nas relações $H=2,5 . L$ e $H=3,0 . L$ ), e oeste e sudeste (na relação $H=3,0 . L$ ), todos os valores de iluminância encontram-se no intervalo suficiente das UDI. Para o céu 12 (claro), para todas as relações $(H=2,0 . L, H=2,5 . L, H=3,0 . L)$, com exceção da orientação da abertura para sul, todos os valores de iluminância encontram-se no intervalo suficiente das UDI. Nas orientações sul, os valores de iluminância encontram-se no intervalo entre 100 Ix e 500 lx, caracterizados como suficientes, mas com necessidade de iluminação complementar.

Constatou-se também que, para o céu 3 (encoberto), céu 7 (parcialmente nublado) e céu 12 (claro), é na variação das relações entre altura de edificação obstruidora $(H)$ e largura de via ( $L$ ) de $H=2,0 . L$ para $H=2,5$. $L$ que ocorrem as maiores taxas de decaimento da iluminância do ambiente interno. No caso do céu 7 (parcialmente nublado) e céu 12 (claro), a influência é ainda maior nas aberturas orientadas para norte. Dessa forma, a atenção deve estar voltada para essa relação de maior taxa de decaimento, visto ser a que acarreta a maior interferência na disponibilidade de iluminação natural no ambiente interno. 
Para o céu 7 (parcialmente nublado) e 12 (claro), constatou-se que a orientação da abertura tem maior influência para a iluminância na altura de edificação obstruidora de $36 \mathrm{~m}$ (relação $H=2$.L), e conforme cresce a relação para $H=2,5 . L$, e $H=3,0$. L vão sendo reduzidas as diferenças de valores entre a maior e a menor iluminância. Dessa forma, conforme aumenta a relação entre a largura de via e a altura da edificação obstruidora, a variação na orientação da abertura passa a ter cada vez menor influência.

Para o céu 3, constatou-se que tanto o aumento da altura da edificação obstruidora nas relações $H=2,0 . L, H=2,5$. L e $H=3,0 . L$ quanto a variação na orientação das aberturas, os valores de iluminância estão em consonância com a NBR5413/82. Porém, para os céus 7 e 12, alguns valores de iluminância encontrados apresentam-se superiores ao estipulado na NBR5413/82. Isso acarretará consequentemente a necessidade do uso de proteções solares, o qual reduzirá a iluminação natural no ambiente interno, com benefícios, porém, na redução do efeito de ofuscamento.

Os resultados obtidos nas simulações confirmam a necessidade de se considerarem as relações da geometria urbana (no caso em questão, a largura de via e as alturas da edificação obstruidora), bem como a orientação das aberturas no estudo da disponibilidade de iluminação no ambiente interno, de forma a garantir as iluminâncias adequadas no ambiente interno, bem como na redução dos gastos com energia elétrica para iluminação artificial.

No que se refere às limitações dos resultados da pesquisa, ressalta-se que as simulações foram realizadas com base na fixação das condições de análise, como a definição da tipologia, área, dimensões e características do fechamento transparente da abertura, bem como das dimensões e superfícies refletoras do ambiente interno. As simulações também limitam a pesquisa ao estudo do ambiente interno localizado na altura equivalente ao quarto pavimento de uma edificação, considerando ser esse o primeiro andar tipo, sendo ainda definidos três tipos de céus para a realização das simulações.

Como perspectiva de continuidade do estudo, prevê-se o refinamento dos resultados por meio da definição de outros pontos de avaliação, o que permitirá análises mais detalhadas do comportamento da iluminância no ambiente interno. Uma das possibilidades é a análise separadamente de cada um dos componentes da iluminação global, parcela direta e parcela refletida, investigando o comportamento de cada uma dessas componentes e as relações entre a largura de via e altura de edificação obstruidora para as orientações analisadas.

Também poderá ser considerada nos estudos futuros a investigação em andares inferiores e superiores ao estudado, bem como a variação dos parâmetros urbanos adotados, objetivando verificar a relevância dessas associações na disponibilidade de iluminação natural no ambiente interno.

\section{Referências}

ARAÚJO, I. A. L.; CABÚS, R. Influência da luz natural refletida pelo entorno na iluminação de edifícios em cânions urbanos no trópico úmido. In: ENCONTRO NACIONAL DE CONFOR- 
TO NO AMBIENTE CONSTRUÍDO, 9; LATINO AMERICANO DE CONFORTO NO AMBIENTE CONSTRUÍDO, 5, 2007, Ouro Preto. Anais... Ouro Preto: Antac, 2007.

ASSOCIAÇÃO BRASILEIRA DE NORMAS TÉCNICAS. NBR 5413: lluminância de interiores. Rio de Janeiro: ABNT, 1992.

ASSOCIAÇÃO BRASILEIRA DE NORMAS TÉCNICAS. NBR 15215-4: Verificação experimental das condições de iluminação interna de edificações - Método de medição. Rio de Janeiro: ABNT, 2005.

CABÚS, R. C. TropLux, versão 6: Guia do Usuário, Maceió: Grilu, 2012.

CABÚS, R. C. Troplux: um sotaque tropical na simulação da luz natural em edificações. In: ENCONTRO NACIONAL DE CONFORTO NO AMBIENTE CONSTRUÍDO, 8.; ENCONTRO LATINO-AMERICANO DE CONFORTO NO AMBIENTE CONSTRUÍDO, 4., 2005, Maceió. Anais... Maceió, AL, 2005.

CAPELUTO, I. G. The influence of the urban environment on the availability of daylighting in office buildings in Israel. Building and Environment, v. 38, n. 5, p. 745-752, May 2003.

COMMISSION INTERNATIONALE L'ACLAIRAGE. Spatial distribution of daylight. Viena: CIE Standard General Sky, 2003.

KRUGER, E.; SUGA, M. Proposta de restrições de altura para cânios urbanos para aproveitamento de luz natural em edificações In: ENCONTRO NACIONAL DE CONFORTO NO AMBIENTE CONSTRUÍDO, 9; LATINO AMERICANO DE CONFORTO NO AMBIENTE CONSTRUÍDO, 5, 2007, Ouro Preto. Anais... Ouro Preto: Antac, 2007.

LARANJA, A. C. Parâmetros urbanos e a disponibilidade de iluminação natural no ambiente interno. 2010. 285f. Tese (Doutorado em Arquitetura) - Universidade Federal do Rio de Janeiro, Programa de Pós-graduação em Arquitetura, Rio de Janeiro.

LARANJA, A. C.; CABÚS, R. C.; GAZZANEO, L. M. C. Interferência da orientação das vias na disponibilidade de iluminação natural do ambiente interno. In: XIV ENCONTRO NACIONAL DE TECNOLOGIA DO AMBIENTE CONSTRUÍDO, 14, 2012, Juiz de Fora. Anais... Juiz de Fora: UFJF, 2012.

LI, D. H. W. et al. A study of the daylighting performance and energy use in heavily obstructed residential buildings via computer simulation techniques. Energy and Buildings, v. 38, n. 11, p. 1343-1348, Nov. 2006.

LITTLEFAIR, P. Daylight, sunlight and solar gain in the urban environment. Solar Energy, v. 70, n. 3, p. 177-185, 2001.

LITTLEFAIR, P. Passive solar urban design: ensuring the penetration of solar energy into the city. Renewable and Sustainable Energy Reviews, v. 2, n. 3, p. 303-326, Sep. 1998

MORAES, O.; SCARAZZATO, P. S. Iluminação natural no meio urbano: estudo de caso com o método dos indicadores de altura admissíveis aplicado a Campinas. In: ENCONTRO NACIONAL SOBRE CONFORTO NO AMBIENTE CONSTRUÍDO, 7. CONFERÊNCIA LATINO-AMERICANA SOBRE CONFORTO E DESEMPENHO ENERGÉTICO DE EDIFICAÇÕES, 3. 2003, Curitiba. Anais... Curitiba, 2003. 
NABIL, A.; MARDALJEVIC, J. Useful daylight illuminances: a replacement for daylight factors. Energy and Buildings, London, v. 38, n. 7, p. 905-913, Jul. 2006.

NG, E. A study of the relationship between daylight performance and height difference of buildings in high density cities using computational simulation. In: INTERNATIONAL BUILDING PERFORMANCE SIMULATION CONFERENCE, 9., 2005, Montreal, Proceedings... Montreal: IBPSA, 2005. p. 847-852.

NIKIFORIADIS, F; PITTS, A. 3D digital geometric reconstruction of the urban environment for daylight simulations studies. In: INTERNATIONAL BUILDING SIMULATION CONFERENCE, 8., Eindhoven, Netherlands, 2003. Proceedings... Eindhoven: IBPSA, 2003.

RATTI, C.; BAKER, N.; STEEMERS, K. Energy consumption and urban texture. Energy and Buildings, London, v. 37, n. 7, p. 762-776, Jul. 2005.

TREGENZA, P.; WATERS, I. M. Daylight coefficients. Lighting Research \& Technology, v. 15, n. 2, p. 65-71, Jun. 1983.

ÜNVER, R. et al. Effect of the facade alternatives on the daylight illuminance in offices. Energy and Building, London, v.35, n. 8, p. 737-746, Sep. 2003.

VITÓRIA (ES). Lei n. ${ }^{\circ} 4821$, de 30 de dezembro de 1998. Institui o Código de Edificações do Município de Vitória e dá outras providências. Prefeitura Municipal de Vitória: Vitória, 1998. Disponível em: <http://www.vitoria.es.gov.br>. Acesso em: 10 julho. 2012.

VITÓRIA (ES). Lei n ${ }^{\circ} 6.705$, de 2006. Dispõe sobre o desenvolvimento urbano no Município de Vitória, institui o Plano Diretor Urbano e dá outras providências. Prefeitura Municipal de Vitória: Vitória, 2006. Disponível em: <http://www.vitoria.es.gov. br>. Acesso em: 11 julho. 2012.

\section{Endereços para correspondências}

Andréa Coelho Laranja

Rua Joaquim Lirio, 96/302 - Praia do Canto

29055-460 - Vitória - ES

andreacoelholaranja@gmail.com

Cristina Engel de Alvarez

Rua Eugenio Netto, 393/602

29055-270 - Vitória - ES

cristina.engel@ufes.br.

Leilani Gomes Correia

Avenida Adolpho Cassoli, 326 - Maruípe

29043-040 - Vitória - ES

leilani.arquitetura@hotmail.com

Ricardo Carvalho Cabús

Rua Dep. Mendonça Braga, 1 - Guaxuma

57038-755 - Maceió - AL

r.cabus@pq.cnpq.br 